\title{
Serological Detection of Antibodies against Anaplasma spp. in Cattle Reared in the Gyeongsangbuk-do, Korea
}

\author{
Min-Goo Seo ${ }^{1,2}$, In-Ouh Ouh², Seung-Hun Lee', Ui-Han Son', Paul John L. Geraldino³, Man Hee Rhee', \\ Oh-Deog Kwon', Tae-Hwan Kim', Dongmi Kwak ${ }^{1,4 *}$ \\ ${ }^{1}$ College of Veterinary Medicine, Kyungpook National University, Daegu 41566, Korea; ${ }^{2}$ Animal and Plant Quarantine Agency, Gimcheon 39660 , \\ Korea; ${ }^{3}$ Department of Biology, School of Arts and Sciences, University of San Carlos, Cebu 6000, Philippines; ${ }^{4}$ Cardiovascular Research Institute, \\ Kyungpook National University, Daegu 41944, Korea
}

\begin{abstract}
Anaplasmosis is a tick-borne, non-contagious, zoonotic disease caused by Anaplasma spp., which include Anaplasma marginale, A. centrale, A. phagocytophilum, A. platys, A. ovis, and A. bovis. Recently, in Korea, the prevalence of Anaplasma spp. has been investigated in some animals, such as dogs, horses, goats, cats, and Korean water deer. In cattle, A. marginale is the most virulent species and regarded as the typical type of species. However, data on the seroprevalence of Anaplasma spp. in cattle in Korea during the last decade is limited. This study was designed to investigate the seroprevalence of bovine anaplasmosis in Korea. From 2010 to 2013, blood samples were collected from 568 cattle. Forty animals (7.0\%) tested seropositive for Anaplasma spp. by cELISA. Despite that current bovine anaplasmosis seropositivity rate in the Gyeongsangbuk-do is lower than those in tropical countries, anaplasmosis needs to be regarded as a concerning disease. The identification of the specific Anaplasma species infecting cattle in this province requires additional molecular studies. Moreover, further monitoring and control programs for bovine anaplasmosis is required, and the information from this study will be beneficial to develop these programs.
\end{abstract}

Key words: Anaplasma, bovine, ELISA, seroprevalence

Anaplasmosis is a tick-borne, infectious, non-contagious, zoonotic disease caused by Anaplasma spp. [1]. Anaplasma spp. are obligate, intracellular, gram-negative organisms with no cell wall. The genus Anaplasma, belonging to the family Anaplasmataceae and order Rickettsiales, comprises Anaplasma marginale, A. centrale, A. phagocytophilum, A. platys, A. ovis, and A. bovis [1]. For instance, A. marginale and A. centrale cause bovine anaplasmosis, and A. ovis infects other domestic and wild ruminant species. In cattle, A. marginale is the most virulent and regarded as the typical type of species; nevertheless, A. marginale does not cause clinical disease in humans [1].

The clinical signs of anaplasmosis are variable, from asymptomatic to fatal [1]. The symptoms of bovine anaplasmosis include anemia, pyrexia, jaundice, depression, anorexia, loss of weight, reduction of milk production, and death [1]. Anaplasmosis occurs worldwide but mainly in tropical and subtropi-

- Received 9 April 2018, revised 30 May 2018, accepted 30 May 2018.

*Corresponding author (dmkwak@knu.ac.kr)

(c) 2018, Korean Society for Parasitology and Tropical Medicine

This is an Open Access article distributed under the terms of the Creative Commons Attribution Non-Commercial License (http://creativecommons.org/licenses/by-nc/4.0) which permits unrestricted non-commercial use, distribution, and reproduction in any

medium, provided the original work is properly cited. cal regions near the equator, and the distribution of Anaplasma spp. seems to be associated with that of ticks [2]. Nevertheless, global warming has driven the expansion of tick habitats, and, thus, of tick-borne diseases.

There are several studies on the detection of A. phagocytophilum in Korea in humans [3], dairy cattle, cats, and horses [4], birds [5], Korean water deer [6], shelter dogs [7], shelter cats [8], and horses [9]. Additionally, there are studies on A. marginale-infected cattle that have been performed by capillary tube agglutination test (CTAT) and ELISA [10] and on A. centrale-infected cattle performed by CTAT [11]. Nevertheless, no information exists on the last decade and on the current status of bovine anaplasmosis in Korea. Therefore, the purpose of this study was to investigate the prevalence of Anaplasma spp. in cattle in Korea, by ELISA.

In 2016, 3,121,169 cattle had been raised in 95,233 farms, 629,441 (20.2\%) of which had been raised in 20,143 (21.2\%) farms in the Gyeongbuk province, Korea [12]. The sample size to be used in the study was determined using the following formula, with an expected disease prevalence of $50 \%$, an accepted absolute error of 5\%, and a confidence level of 95\%, with a simple random sampling design [13]: 


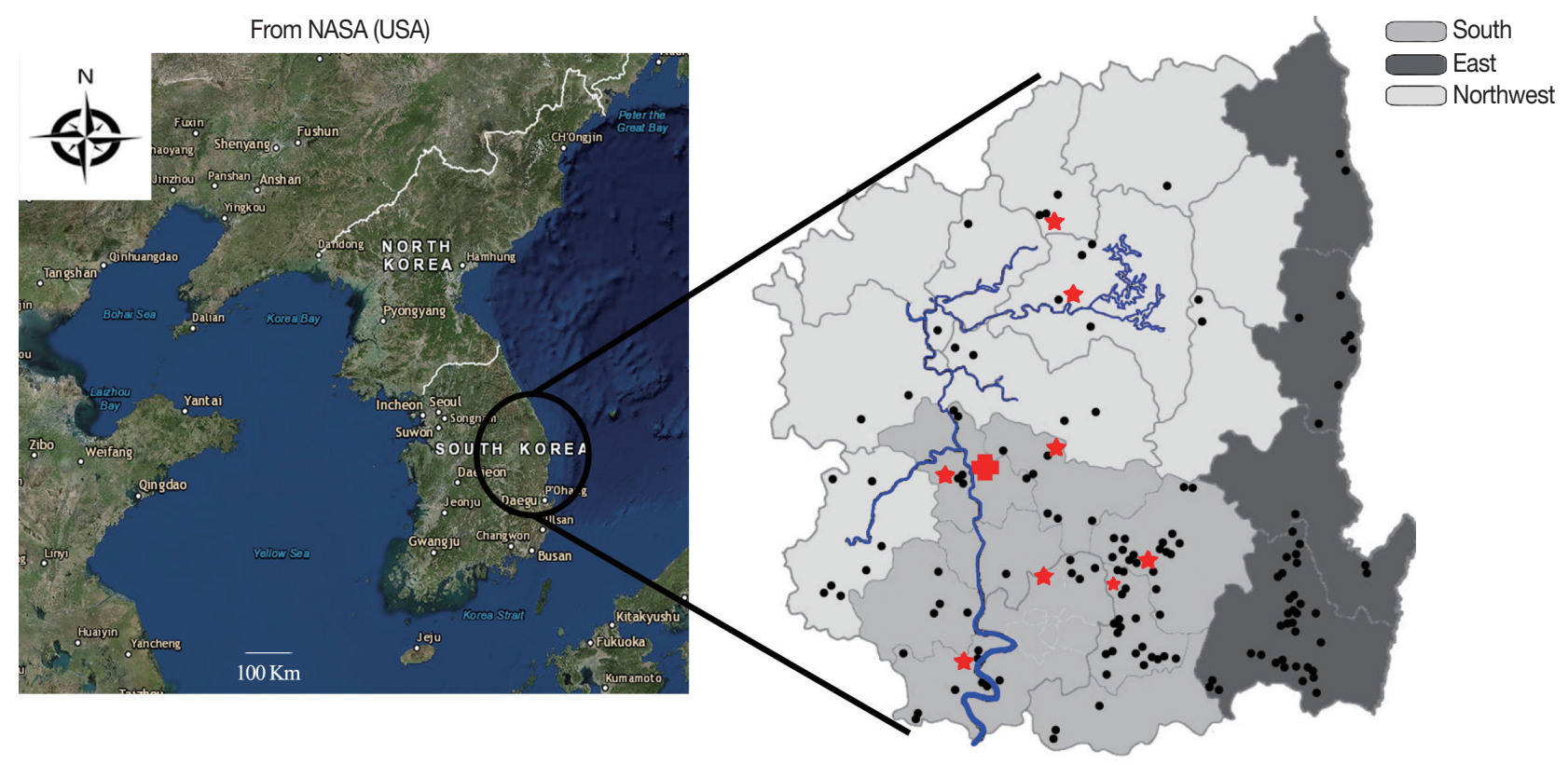

Fig. 1. Provincial map of Gyeongbuk, Korea, divided in 3 administrative districts. Samples collected from cattle farms are denoted by dots; the Haepyeong habitat for migratory birds is denoted by a red cross; local butcheries are denoted by red stars; and the Nakdong River is denoted by blue color.

$$
n=\frac{1.96^{2} p_{\exp }\left(1-p_{\exp }\right)}{d^{2}}
$$

where $n=$ required sample size, $p_{\exp }=$ expected prevalence, and $d=$ desired absolute precision.

According to the formula, a minimum of 384 samples were required. In this study, 568 animals were randomly selected in the Gyeongbuk province, in 2016 (Fig. 1).

The study area was divided into 3 regions by administrative districts, most of the areas being mountainous: the Northwestern region, which has mountains; the Eastern region, which has coast; and the Southern region, which is an urban and suburban area with lowland plains. The Nakdong River runs from the Northwestern to the Southern region, where a riverine marshland named Haepyeong, a habitat for migratory birds, is located (Fig. 1).

From 2010 to 2013, blood samples were collected from 568 cattle reared in the Gyeongbuk province. Sera were stored at $-20^{\circ} \mathrm{C}$ until the analysis was performed. For the statistical analysis, the age and sex of the cattle and the location of the farm from which the samples had been collected were recorded (Table 1).

To detect antibodies against Anaplasma spp., a commercial Anaplasma antibody cELISA kit (VMRD, Pullman, Washington, USA), using recombinant major surface protein 5 to detect $A$.
Table 1. Seroprevalence of Anaplasma spp. in 568 cattle in the Gyeongsangbuk-do as revealed by cELISA results, according to sex, age, and region

\begin{tabular}{lcccc}
\hline Group & No. tested & No. positive (\%) & $95 \% \mathrm{Cl}$ & $P$-value \\
\hline Sex & & & & \\
$\quad$ Male & 22 & $3(13.6)$ & $0-28.0$ & $<0.001$ \\
$\quad$ Female & 74 & $15(20.3)$ & $11.1-29.4$ & \\
$\quad$ Castrated & 472 & $22(4.7)$ & $2.8-6.6$ & \\
Age & & & & \\
$\quad$ Young ( $\leq 1$ y) & 37 & $4(10.8)$ & $0.8-20.8$ & $<0.001$ \\
Adult (2 y) & 474 & $21(4.4)$ & $2.6-6.3$ & \\
Old ( $\geq 3$ y) & 57 & $15(26.3)$ & $14.9-37.7$ & \\
Region & & & & \\
$\quad$ East & 155 & $5(3.2)$ & $0.4-6.0$ & 0.009 \\
$\quad$ Northwest & 124 & $7(5.6)$ & $1.6-9.7$ & \\
$\quad$ South & 289 & $28(9.7)$ & $6.3-13.1$ & \\
Total & 568 & $40(7.0)$ & $4.9-9.2$ & \\
\hline
\end{tabular}

$\mathrm{Cl}$, confidence interval.

marginale, A. ovis, and A. centrale in bovine serum, was used, following the manufacturer's instruction. The percentage of inhibition was calculated for each sample as follows: Value $(\%)=100 \times\left[1-\left(\mathrm{OD}_{\text {sample }} / \mathrm{OD}_{\text {negative control }}\right)\right]$. Samples with a percentage greater than $30 \%$ were considered positive; those with $30 \%$ or less were considered negative.

Fisher's exact test and linear-by-linear association were conducted to analyze statistical differences among the groups, us- 
ing SPSS 21.0 software (IBM, Armonk, New York, USA). Due to the difference in group sizes, the Bonferroni post hoc method was performed [14]. A P-value below 0.05 was regarded as statistically significant. The $95 \%$ confidence interval was also calculated.

Forty out of $568(7.0 \%)$ animals tested seropositive for Anaplasma spp. (Table 1). According to the sex, 3 of 22 males (13.6\%), 15 of 74 females (20.3\%), and 22 of 472 castrated (4.7\%) cattle were seropositive. By age, 4 samples of 37 (10.8\%) from calves under 1 year old, 21 samples of 474 (4.4\%) from 2-year-old animals, and 15 samples of 57 (26.3\%) from 3-year-old or older animals were seropositive. Regarding the region, 5 of 155 (3.2\%) from the East, 28 of 289 (9.7\%) from the South, and 7 of 124 (5.6\%) from the Northwest were seropositive. Statistical differences in seroprevalence were observed in age, sex, and region $(P<0.05)$.

In this study, cELISA was performed to identify antibodies against Anaplasma spp. in sera from cattle reared in the Gyeongbuk province. This method is a good choice for the serological survey of bovine anaplasmosis, including infection by A. marginale, because of its sensitivity (96.0\%), specificity (95.2\%), and low cost [15]. In this study, 7.0\% of the animals tested were seropositive for Anaplasma spp. There were several studies in which the detection of Anaplasma spp. has been performed by ELISA, such as that in cattle in Iran $(8 / 105,7.6 \%)$ [16], cattle in Poland $(23 / 1,326,1.73 \%)$ [17], dairy cattle in Puerto Rico $(743 / 2,414$, 27.4\%) [18], cattle and buffalo in North India (47/184, 31.7\%) [19], cattle in USA (15.02\%) [15], and goats in Korea (36/544, 6.6\%) [20]. The overall seropositivity result obtained herein $(7.0 \%)$ is quite lower than those in other studies but similar to that in Iran [16]. The countries showing high infection rates are located in tropical and subtropical regions; Iran is a region of highlands and savannas, and the Korean peninsula is not yet a subtropical climate. Therefore, the climate of the region seems to affect Anaplasma spp. infection rate.

Sorting the results by the sex of the animals, females presented the highest seropositivity (20.3\%), followed by males (13.6\%) and castrated cattle (4.7\%), which showed the lowest value. According to previous gender-wise studies in tick-borne diseases, females are preferred hosts over males [21]. Regarding age, our study showed that 3-year-old or older animals presented a higher rate of seropositivity to anaplasmosis (26.3\%) than calves (10.8\%) and 2-year-old animals (4.4\%). It seems that age affects the serum levels of antibodies against Anaplas$m a$ spp. This tendency in the age-related seroprevalence was similar to that observed in previous studies $[1,16,19]$.
Regarding the results by region, the Southern area showed the highest seroprevalence $(9.7 \%)$, followed by the Northwestern (5.6\%) and the Eastern regions (3.2\%). In Texas, a study performed on cattle on several local auction markets showed a close relationship between the region and the serological level of anaplasmosis, with the national and international herd movement increasing the potential exposure to ticks [15]. In the present study, 6 of the 8 butcheries were located in the Southern region, whereas the other 2 butcheries were located in the Northwestern region. They are in the center of the national cattle herd movement, which, similarly to the auction markets of Texas, may have effects on the serological results.

The riverside is a good habitat for ticks [22]. In this study, the riverine marshland located in the Southern region is a shelter for migratory birds travelling northward from Jeju Island to the mainland of Korea. Birds have a high potential for introducing infected immature ticks to distant areas [5]; therefore, this means that the movement of birds near the Haepyeong habitat can affect the seroprevalence of bovine anaplasmosis in this study area. Nowadays, the ticks' habitat area is expanding due to global temperature change [2], and tickborne diseases, usually endemic to tropical regions, are gradually affecting areas of cold climates [23]. The number of reports of tick-borne diseases is increasing, and the areas of endemic regions in China are expanding [24]. These diseases can ultimately affect not only livestock but also humans; thus, more preventive efforts are required. The data obtained in this study would be useful for epidemic assays and prevention of sporadic infections in areas other than endemically affected regions.

Until now, it was difficult to access information on bovine anaplasmosis in Korea. We found that seroprevalence of Anaplasma spp. in cattle is relatively low in Korea. Nevertheless, the result shows that cattle is infected with Anaplasma spp. Identification of the specific species, A. marginale, A. ovis, A. bovis, A. phagocytophilum, and $A$. centrale, requires further tests, such as PCR and phylogenetic analysis. The current seropositivity rate of bovine anaplasmosis in the Gyeongsangbuk-do indicates that it is not yet a dominant tick-borne disease in cattle, unlike tropical countries; however, it needs to be regarded as a concerning disease. Therefore, continuous monitoring and control programs for bovine anaplasmosis are required, and the information from this study may be beneficial to establish such programs. 


\section{ACKNOWLEDGMENT}

This research was supported by Kyungpook National University Bokhyeon Research Fund, 2015.

\section{CONFLICT OF INTEREST}

We declare that we have no conflict of interest related to this work.

\section{REFERENCES}

1. Aubry P, Geale DW. A review of bovine anaplasmosis. Transbound Emerg Dis 2011; 58: 1-30.

2. Kocan KM, de la Fuente J, Blouin EF, Coetzee JF, Ewing SA. The natural history of Anaplasma marginale. Vet Parasitol 2010; 167: 95-107.

3. Heo EJ, Park JH, Koo JR, Park MS, Park MY, Dumler JS, Chae JS. Serologic and molecular detection of Ehrlichia chaffeensis and Anaplasma phagocytophila (human granulocytic ehrlichiosis agent) in Korean patients. J Clin Microbiol 2002; 40: 3082-3085.

4. Chae JS, Heo EJ, Park JH, Choi KS, Dumler JS, Lee SS, Kang TY, Yang JH, Kim DY, Kim JG, Choi GC, Kang MI. Detection of antibodies reacting with Anaplasma phagocytophilum and Ehrlichia chaffeensis from cats, horses and cattle in Korea. J Vet Clin 2009; 26: 515-519.

5. Oh MR, Moon KH, Kim SY, Kim YG, Choi CY, Kang CW, Kim HJ, Lee KK, Yun YM. Prevalence of Anaplasma sp. in Thrushes (Family Turdidae) in Jeju Island, Republic of Korea. J Vet Clin 2014; 31: 206-211.

6. Kang JG, Ko S, Kim YJ, Yang HJ, Lee H, Shin NS, Choi KS, Chae JS. New genetic variants of Anaplasma phagocytophilum and Anaplasma bovis from Korean water deer (Hydropotes inermis argyropus). Vector Borne Zoonotic Dis 2011; 11: 929-938.

7. Lee S, Lee SH, VanBik D, Kim NH, Kim KT, Goo YK, Rhee MH, Kwon OD, Kwak D. First molecular detection and phylogenetic analysis of Anaplasma phagocytophilum in shelter dogs in Seoul, Korea. Ticks Tick Borne Dis 2016; 7: 945-950.

8. Lee SH, VanBik D, Kim NH, Park SJ, Kwon OD, Kim TH, Kwak D. First molecular detection and genetic analysis of Anaplasma phagocytophilum in shelter cats in Seoul, Korea. Infect Genet Evol 2016; 46: 71-73.

9. Lee SH, Kim KT, Yun SH, Choi E, Lee GH, Park YS, Cho KH, Yi S, Kwon OD, Kim TH, Kwak D. Serological and molecular detection of Anaplasma phagocytophilum in horses reared in Korea. Vet Med 2015; 60: 533-538.

10. Lee JM, Kwon OD, Song HJ, Park JH, Choi KS. Anaplasma margi- nale infection in holstein calves during winter. Korean J Vet Res 1997; 37: 911-916 (in Korean).

11. Jeon Y. Isolation of Anaplasma centrale from Korean cattle. Korean J Vet Res 1978; 18: 19-22 (in Korean).

12. Korean Statistical Information Service. Number of farms in households and animals in heads by type; the fourth quarter of 2016. [Internet]; 2016. Available from: http://kosis.kr/statHtml/statHtml do?orgId=101\&tblId=DT_1EO099\&conn_path=I2\&language=en.

13. Thrusfield MV. Veterinary Epidemiology. 3rd ed. Oxford, UK. Blackwell Science. 2005, pp 228-330.

14. Dunn OJ. Multiple comparisons among means. J Am Stat Assoc 1961; 56: 52-64.

15. Hairgrove TB, Craig TM, Budke CM, Rodgers SJ, Gill RJ. Seroprevalence of Anaplasma marginale in Texas cattle. Prev Vet Med 2014; 116: 188-192.

16. Khezri M. Seroprevalence of Anaplasma infection in sheep and cattle in Kurdistan province of Iran with an overview of one decades of its epidemiological status in Iran. JSRR 2015; 6: 26-36.

17. Szweda W, Siemionek J, Lipińska E, Błaszczak U, Michałowska M. Prevalence survey of Anaplasma marginale infection in cattle in the Warmia and Mazury region of Poland by competitive ELISA MSP-5. Med Weter 2011; 67: 838-842 (in Polish).

18. Urdaz-Rodriguez JH, Fosgate GT, Alleman AR, Rae DO, Donovan GA, Melendez P. Seroprevalence estimation and management factors associated with high herd seropositivity for Anaplasma marginale in commercial dairy farms of Puerto Rico. Trop Anim Health Prod 2009; 41: 1439-1448.

19. Sharma A, Singla LD, Kaur P, Bal MS. PCR and ELISA vis-à-vis microscopy for detection of bovine anaplasmosis: a study on associated risk of an upcoming problem in North India. ScientificWorldJournal 2015; 2015: 352519.

20. Lee SH, Jung BY, Kwak D. Evidence of Anaplasma spp. exposure in native Korean goats (Capra hircus coreanae). Vet Med 2015; 60: 248-252.

21. Rather SA, Tak H, Kakru DK. Seroprevalence of Babesia bigemina and Anaplasma marginale in domestic animals of district Ganderbal. Sci J Vet Adv 2016; 5: 74-79.

22. Guerra M, Walker E, Jones C, Paskewitz S, Cortinas MR, Stancil A, Beck L, Bobo M, Kitron U. Predicting the risk of Lyme disease: habitat suitability for Ixodes scapularis in the north central United States. Emerg Infect Dis 2002; 8: 289-297.

23. Walker DH. Changing dynamics of human-rickettsial interactions. Am J Trop Med Hyg 2016; 94: 3-4.

24. Fang LQ, Liu K, Li XL, Liang S, Yang Y, Yao HW, Sun RX, Sun Y, Chen WJ, Zuo SQ, Ma MJ, Li H, Jiang JF, Liu W, Yang XF, Gray GC, Krause PJ, Cao WC. Emerging tick-borne infections in mainland China: an increasing public health threat. Lancet Infect Dis 2015; 15: 1467-1479. 